\title{
Resonance fluorescence of a site-controlled quantum dot realized by the buried-stressor growth technique
}

Cite as: Appl. Phys. Lett. 110, 111101 (2017); https://doi.org/10.1063/1.4978428

Submitted: 26 December 2016 . Accepted: 27 February 2017 . Published Online: 13 March 2017

Max Strauß, Arsenty Kaganskiy, Robert Voigt, Peter Schnauber (D), Jan-Hindrik Schulze, Sven Rodt, André

Strittmatter, and Stephan Reitzenstein (i)
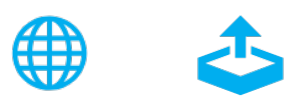

View Online

\section{ARTICLES YOU MAY BE INTERESTED IN}

Electrically driven and electrically tunable quantum light sources

Applied Physics Letters 110, 071102 (2017); https://doi.org/10.1063/1.4976197

Excitonic lasing of strain-free InP(As) quantum dots in AllnAs microdisk Applied Physics Letters 110, 121101 (2017); https://doi.org/10.1063/1.4979029

Micropillars with a controlled number of site-controlled quantum dots

Applied Physics Letters 112, 071101 (2018); https://doi.org/10.1063/1.5017692

Lock-in Amplifiers Find out more today

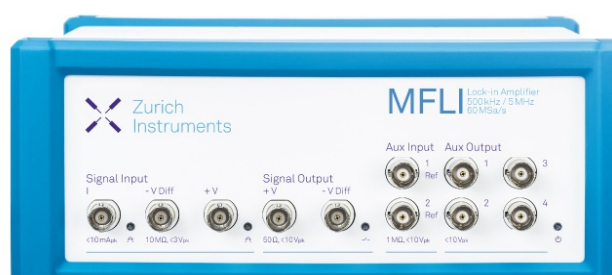

Zurich - Instruments 


\title{
Resonance fluorescence of a site-controlled quantum dot realized by the buried-stressor growth technique
}

\author{
Max Strauß, Arsenty Kaganskiy, Robert Voigt, Peter Schnauber, Jan-Hindrik Schulze, \\ Sven Rodt, André Strittmatter, ${ }^{\text {a) }}$ and Stephan Reitzenstein ${ }^{\text {b) }}$ \\ Institut für Festkörperphysik, Technische Universität Berlin, 10623 Berlin, Germany
}

(Received 26 December 2016; accepted 27 February 2017; published online 13 March 2017)

\begin{abstract}
Site-controlled growth of semiconductor quantum dots (QDs) represents a major advancement to achieve scalable quantum technology platforms. One immediate benefit is the deterministic integration of quantum emitters into optical microcavities. However, site-controlled growth of QDs is usually achieved at the cost of reduced optical quality. Here, we show that the buried-stressor growth technique enables the realization of high-quality site-controlled QDs with attractive optical and quantum optical properties. This is evidenced by performing excitation power dependent resonance fluorescence experiments at cryogenic temperatures showing QD emission linewidths down to $10 \mu \mathrm{eV}$. Resonant excitation leads to the observation of the Mollow triplet under CW excitation and enables coherent state preparation under pulsed excitation. Under resonant $\pi$-pulse excitation we observe clean single-photon emission associated with $g^{(2)}(0)=0.12$ limited by non-ideal laser suppression. Published by AIP Publishing. [http://dx.doi.org/10.1063/1.4978428]
\end{abstract}

Quantum emitters are central objects in emerging quantum technologies. ${ }^{1}$ Quantum communication, for instance, is based on single photons as information carriers. ${ }^{2}$ While simple quantum key distribution can be implemented by strongly attenuated lasers, advanced schemes for long distance quantum communication rely on entanglement distribution which requires real quantum emitters with a non-classical photon statistics, high photon indistinguishably and high extraction efficiency. ${ }^{3}$ Recent experiments have shown that selfassembled semiconductor quantum dots (QDs) are prime candidates to meet these requirements. ${ }^{4}$ Moreover, in contrast to non-classical light sources relying on spontaneous parametric down conversion, ${ }^{5}$ QDs offer the great prospect of providing single photons on demand. ${ }^{4}$ The down-side of growing QDs by self-assembly is randomness in position and emission energy. This is particularly problematic when it comes to device integration. As a result, in-situ lithography techniques were invented to circumvent this issue by pre-selecting suitable QDs from a large ensemble. ${ }^{6,7}$ On the other hand, onchip schemes for photonic computing are usually based on regular arrays of coupled single photon emitters, or on quantum emitters integrated into regular waveguides. ${ }^{8,9}$

In order to facilitate scalable device concepts based on QDs, different schemes for their site-controlled growth have been developed. Prominent examples apply arrays of etched nanoholes or inverted pyramids as nucleation centers for the localized growth of QDs. ${ }^{10-13}$ These approaches lead to excellent site-control of the QDs position and allows for the device integration of spatially aligned single QDs. However, tight site-control goes along with enhanced impact of defect centers and non-radiative recombination when the QDs form in close proximity to the etched nanoholes. ${ }^{14}$ Indeed, there is a general trade-off between

\footnotetext{
${ }^{a)}$ Present address: Abteilung für Halbleiterepitaxie, Otto-von-Guericke Universität, 39106 Magdeburg, Germany.

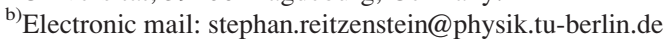

site-selectivity and optical quality of site-controlled quantum dots (SCQDs). ${ }^{15,16}$ Other approaches based on SCQDs in non-planar sample geometries such as QDs embedded in nanowires have shown promise regarding the optical quality of the QDs, ${ }^{17}$ but offer challenges regarding their scalability and integrability. Another promising technology platform for the realization of site-controlled QDs in planar sample geometries is based on a buried stressor. ${ }^{18,19}$ In this approach, strain-tuning by a oxide-aperture leads to the localized formation of QDs where the number of site-controlled QDs can be controlled by the diameter of the aperture. ${ }^{19,20}$ This approach provides a pristine growth surface fairly separated from the stressor and, thus, promises high optical quality of the localized QDs.

In this letter, we report on the optical and quantum optical properties of single QDs grown on a buried-stressor under strict resonant excitation. We observe the Mollow triplet under cw-excitation allowing us to identify exciton-phonon coupling as dominant dephasing mechanism in our system. Furthermore, by using resonant pulsed excitation to deterministically prepare inversion of our two-level system, we are able to demonstrate single-photon emission $\left(g^{(2)}(0)=0.12\right)$.

The SCQDs were grown via metal-organic chemical vapor deposition (MOCVD) using an n-doped GaAs substrate. The fabrication process starts with the growth of a template for subsequent etching of mesa-structures and oxidation of local apertures. ${ }^{19}$ The layer structure comprises a distributed Bragg reflector (DBR) mirror consisting of 27 pairs of $\lambda / 4$ thick $\mathrm{Al}_{0.90} \mathrm{Ga}_{0.10} \mathrm{As} / \mathrm{GaAs}$ followed by a $30 \mathrm{~nm}$ thick AlAs layer embedded into $40 \mathrm{~nm}$ thick AlGaAs claddings. Following the initial growth step, 20-21 $\mu \mathrm{m}$ wide and square shaped mesas are formed via reactive ion etching in an inductively coupled plasma (ICP-RIE). The square shape of the mesas is chosen so as to minimize the formation of defects during overgrowth which tend to form faster on side walls in [110] and [1-10] directions. This etching removes the semiconductor material down to the lowest mirror pair to 
laterally expose the AlAs layer for oxidation performed at $420{ }^{\circ} \mathrm{C}$ in a $\mathrm{H}_{2} \mathrm{O} / \mathrm{N}_{2}$ atmosphere. Here, in-situ optical monitoring of the oxidation process facilitates controlling the aperture diameter on the scale of a few hundred nanometers. Afterwards, the oxidized mesas are overgrown by a $50 \mathrm{~nm}$ thick GaAs buffer layer followed by the site-controlled InGaAs QDs sandwiched between the oxide aperture and an upper AlGaAs charge-carrier antidiffusion barrier and a thin (30 nm) GaAs capping layer (cf. Fig. 1(a)).

Our site-controlled growth is achieved by the local modification of the free energy of a GaAs (001) surface using spatially modulated strain fields caused by a partial oxidation of the underlying AlAs layer. A crucial parameter hereby is the aperture diameter which influences the strain distribution. ${ }^{19} \mathrm{At}$ aperture diameters close to $500 \mathrm{~nm}$, the subsequent growth of the strained InGaAs layer preferentially takes place at the local tensile strain maximum directly on top of the AlAs aperture located in the middle of the mesas. Consequently, single QDs can be positioned in the middle of the mesas as is shown exemplary in the atomic force microscope image in Fig. 1(b), where 2 QDs are positioned central to the aperture with a diameter of $\approx 700 \mathrm{~nm}$. This diameter also gives the upper bound of the alignment accuracy of SCQDs in the center of the larger mesa structures. Even though this alignment accuracy is lower than for SCQDs based on nanohole arrays, it is well suited for spatially deterministic integration of QDs into resonant-cavity light emitting diodes ${ }^{18}$ and into micropillar cavities with a few $\mu \mathrm{m}$ diameter and a comparatively large lateral mode profile. Statistical analysis shows that the probability of site-controlled nucleation, i.e., nucleation of a QD on top of the aperture as opposed to unwanted nucleation next to the aperture, is around $65 \%$ measured over an area of $5 \mu \mathrm{m} \times 5 \mu \mathrm{m}$ relevant for device processing of, e.g., micropillar cavities.

The sample was mounted inside a helium flow cryostat and cooled to $5 \mathrm{~K}$. A linearly polarized beam of a tunable laser is focused onto the sample surface using a microscope objective $(\mathrm{NA}=0.65)$, which also collects the resultant fluorescence emitted by the QDs. Polarization suppression of the laser with an extinction ratio of more than 6 orders of magnitude is achieved by using a combination of a $\lambda / 4$-plate and two polarizing beam splitters. Ultimately, the fluorescence light is coupled into a single mode fiber for detection. Depending on the experimental requirements, the fiber is either directly connected to a single photon counting module (SPCM) or to a grating spectrometer with a spectral resolution of $25 \mu \mathrm{eV}$. For reference measurements involving non-resonant excitation we use a laser diode emitting at $785 \mathrm{~nm}$.
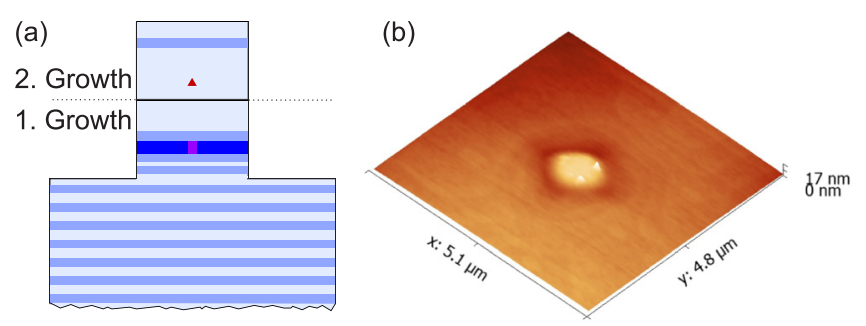

FIG. 1. (a) Schematic view of a fully processed and overgrown structure with SCQD. (b) Atomic force microscopy (AFM) image of two site-controlled quantum dots positioned over a buried stressor with an aperture diameter of $\approx 700 \mathrm{~nm}$.
We pre-characterize the sample by spatially mapping the photoluminescence emitted from QDs under nonresonant excitation (spectral range of plotted intensity: 920-926 nm). A typical $\mu$ PL map scan of a mesa with an oxide-aperture diameter of $700 \mathrm{~nm}$ is presented in Fig. 2. In addition to ensemble emission along the edges of the mesa with the size of $20 \mu \mathrm{m}$ (side-length), we identify five distinct emission centers associated with QDs. The brightest emission originates from a SCQD close to the center of the mesa structure and aligned to the oxide aperture. Interestingly, the QD emission is spatially correlated to a local minimum of the wetting layer emission on top of the oxide aperture (cf. Fig. 2(b)). In general, we use this type of mapping to visualize the (approximate) position of the oxide-aperture in the pre-characterization step. Fig. 2(c) shows a $\mu \mathrm{PL}$ spectrum of the target $\mathrm{QD}$ under non-resonant excitation at $\mathrm{P}=1.4 \mu \mathrm{W}$. The dominating line is identified as neutral exciton and has an emission linewidth of $45 \mu \mathrm{eV}$.

Next, we sweep the tunable resonant laser across the transition indicated in Fig. 2(c) and record the emitted fluorescence with a SPCM. A typical excitation spectrum at $240 \mathrm{nW}$ is shown in Fig. 2(d). The linewidth for excitation powers below $10 \mathrm{nW}$ is about $10 \mu \mathrm{eV}$ (cf. Fig. 2(e)) which indicates the non-Fourier limited photon emission. It is still one of the smallest values reported so far for SCQDs which supports the appealing prospects of the buried stressor approach. The emission linewidth could possibly be further improved by either using electrical fields to control the charge environment of the $\mathrm{QD}^{21}$ or by reduction of lifetime of the emitter via a cavity. ${ }^{22}$ The observed two lines correspond most likely to the finestructure splitting of the exciton $\left(\Delta E_{\mathrm{FSS}}=18.5 \mu \mathrm{eV}\right)$. The finestructure splitting could be manipulated using piezo strain tuning. This technique has been used to suppress the finestructure splitting for generating entangled photon pairs ${ }^{23}$ and is compatible with our planar sample geometry. Increasing the power of the resonant laser we observe the typical saturation behaviour of a two-level system (cf. Fig. 2(f)). Fitting the data with $I=\mathrm{I}_{\infty} \mathrm{P} /\left(\mathrm{P}+\mathrm{P}_{\text {sat }}\right)$ where $\mathrm{P}$ is the laser power, $\mathrm{P}_{\text {sat }}$ the laser power at saturation, and $\mathrm{I}_{\infty}$ the maximum fluorescence intensity, we obtain the laser power at a saturation of $\mathrm{P}_{\text {sat }}=89$ nW.

In order to prove that we can coherently drive the target SCQD, we perform excitation power dependent resonance fluorescence spectroscopy. The coherent interaction between the light field and the QD exciton is reflected in the appearance of a Mollow triplet in the emission spectrum under strong resonant excitation. At an excitation power of $260 \mu \mathrm{W}$ (cf. Fig. 3(a)), the ratio between the sidepeak area and the central peak area is around 1:3, i.e., larger than the expected ratio of $1: 2$, which is probably caused by imperfect laser suppression as well as pure dephasing typical of solid state systems. We fit the sum of three independent Voigts to the spectrum fixing the linewidth of the Gaussian contribution to the resolution limit of our spectrometer $(25 \mu \mathrm{eV})$. Observing the spectra as a function of the laser power incident on the quantum dot (see Fig. 3(b)) reproduces the expected linear dependence of the F- and T-line on the amplitude of the electric field: the F- and T-line shift by $(-4.78 \pm 0.02) \mu \mathrm{eV} / \sqrt{\mu \mathrm{W}}$ and $(5.12 \pm 0.04) \mu \mathrm{eV} / \sqrt{\mu \mathrm{W}}$, respectively. The full width at half maximum (FWHM) of the sidepeaks is given by 

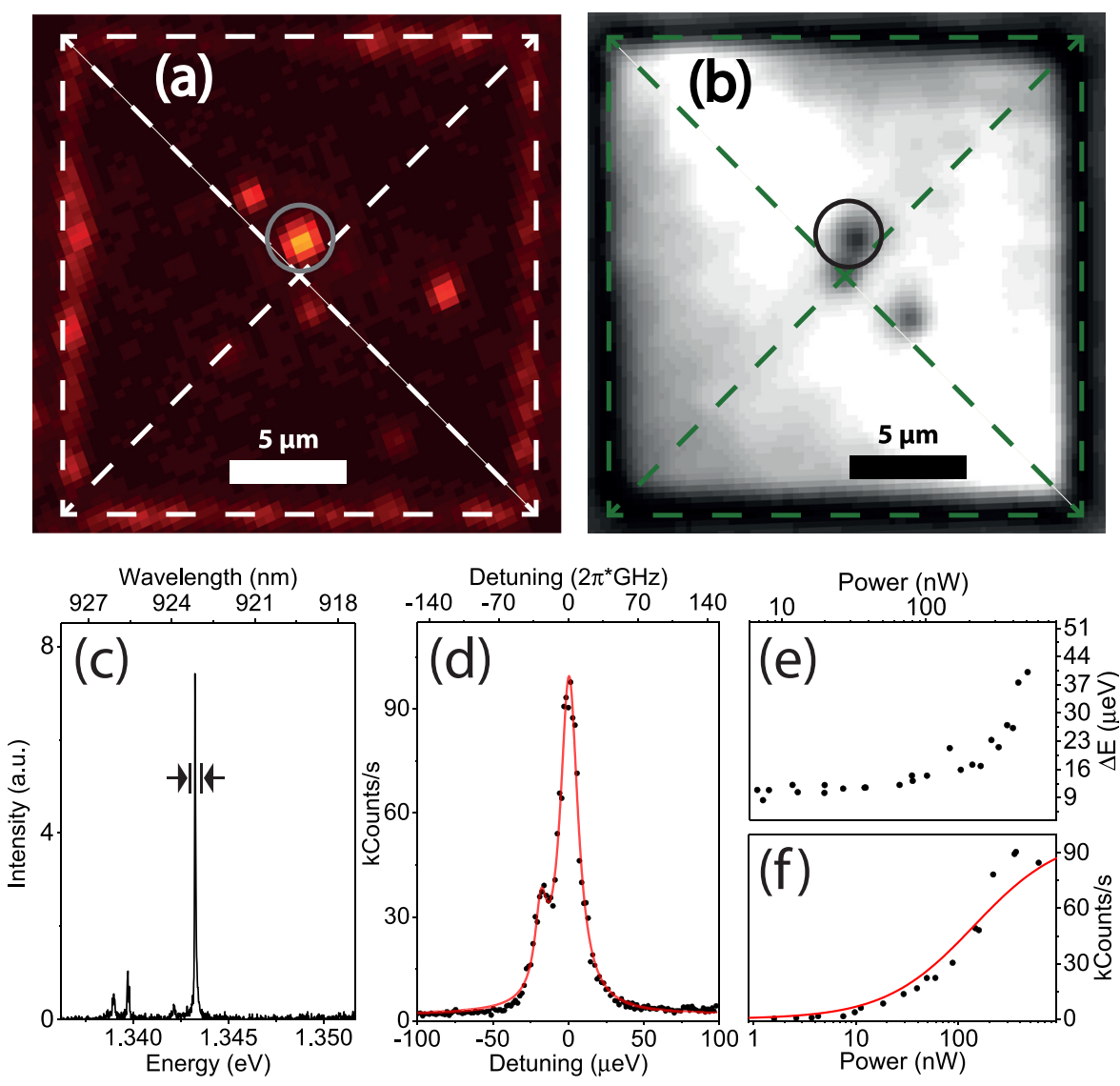

FIG. 2. (a) $2 \mathrm{D} \mu \mathrm{PL}$ intensity map of a mesa containing a SCQD (marked by a circle) very close to its center. (b) $2 \mathrm{D}$ cathodoluminescence intensity map of the same mesa where the intensity is obtained by integrating over the emission from the wetting layer (wavelength interval 900-915 nm). The position of the SCQD under study nicely correlates with the area of reduced wetting layer emission intensity, also indicated by a circle. (c) Non-resonant $\mu \mathrm{PL}$ spectrum of the QD under investigation with an excitonic (X) linewidth (FWHM) of $45 \mu \mathrm{eV}$. (d) Resonance scan of the tunable laser across the $\mathrm{X}$ transition. (e) Linewidth dependence on the excitation power. (f) Excitation power dependent resonance fluorescence intensity of the $\mathrm{X}$ transition. All spectra were taken at $5 \mathrm{~K}$.

$$
\Delta \omega=\frac{3}{2} \Gamma_{1}+\gamma_{\mathrm{PD}}+\gamma_{0},
$$

where $\Gamma_{1}$ is the spontaneous radiative emission rate, $\gamma_{\mathrm{PD}}$ is the phonon-induced pure dephasing rate, and $\gamma_{0}$ is the nonphonon induced dephasing rate. ${ }^{24}$ Accordingly, the sidepeaks have been shown to be particularly susceptible to dephasing effects and can thus be used to identify and characterize the dephasing mechanisms. ${ }^{16,24,25}$ The extracted linewidths of the sidepeaks as a function of the laser power are shown in Fig. 3(c). The linear increase observed in our experiment is consistent with pure dephasing induced by longitudinal acoustic (LA) phonons. Furthermore, using the y-axis intersection and the independently measured lifetime of our emitter $T_{1}$ $=\frac{1}{\Gamma_{1}}=(898 \pm 41) \mathrm{ps}$ (measurement not shown), we obtain a non-phonon induced dephasing rate of $\gamma_{0}=(6.0 \pm 1.3) \mu \mathrm{eV}$ for vanishing pump power (i.e., $\gamma_{\mathrm{PD}}=0$ ).

To demonstrate the quantum nature of emission, we perform photon statistics measurements of our site-controlled QDs under pulsed resonant excitation. For this purpose, we use a mode locked Ti:Sapphire laser providing pulses with a temporal width of $2 \mathrm{ps}$ (FWHM) at a repetition rate of $80 \mathrm{MHz}$. A typical spectrum of the QD under pulsed excitation is shown in Fig. 4(a) for an excitation power of $1 \mu \mathrm{W}$. The single-line spectrum is very clean, and the remaining background around the QD transition is attributed to imperfect laser suppression.

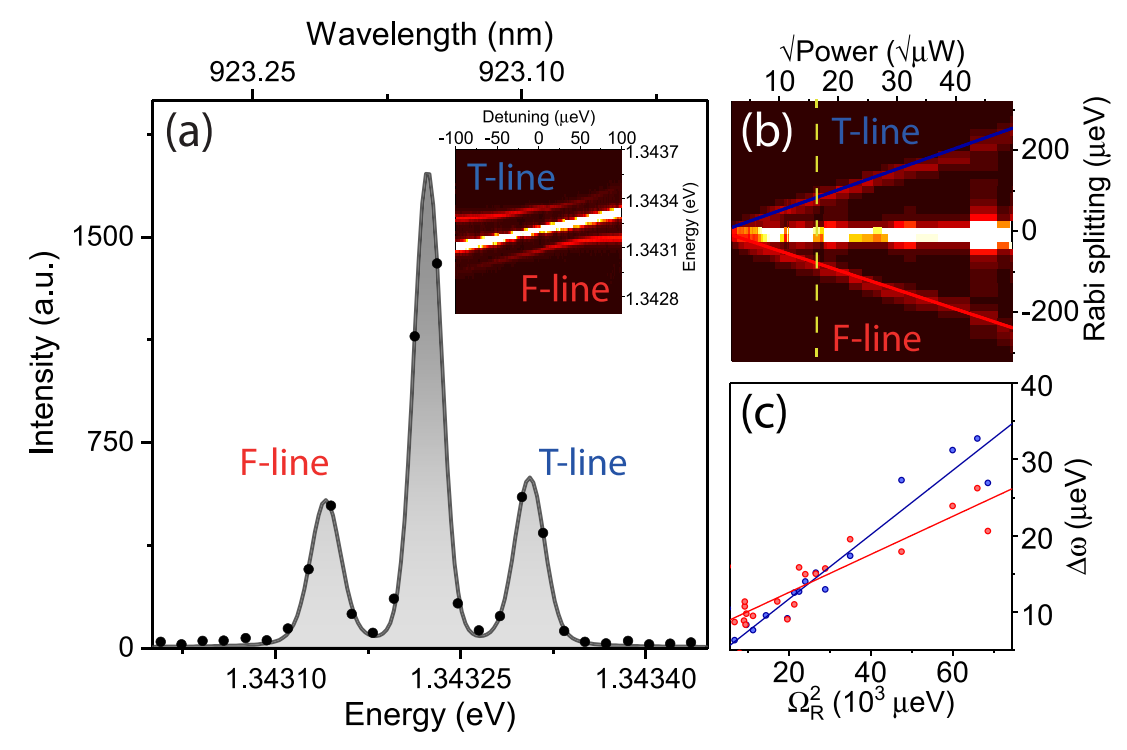

FIG. 3. (a) Emission spectrum of the target quantum dot under strong coherent excitation with $\mathrm{P}=260 \mu \mathrm{W}$ (indicated by the dashed vertical (yellow) line in (b)). The characteristic Mollowtriplet evidences the coherent lightmatter interaction under a strong pump. Inset: Dispersive behaviour of the emission spectrum by tuning the laser across the resonance of the bare quantum dot. (b) Emission spectrum as a function of the square root of the excitation power. The Rabi splitting increases linearly with a slope of $5.1(-4.8) \mu \mathrm{eV} / \sqrt{\mu \mathrm{W}}$. (c) Blue (red) dots: linewidth (FWHM) of the $\mathrm{T}(\mathrm{F})$-line as a function of excitation power. A linear function $\Delta \omega \propto$ $\chi \Omega_{R}^{2}$ is fit to the data giving a slope of $\chi=0.42(0.26)$. 


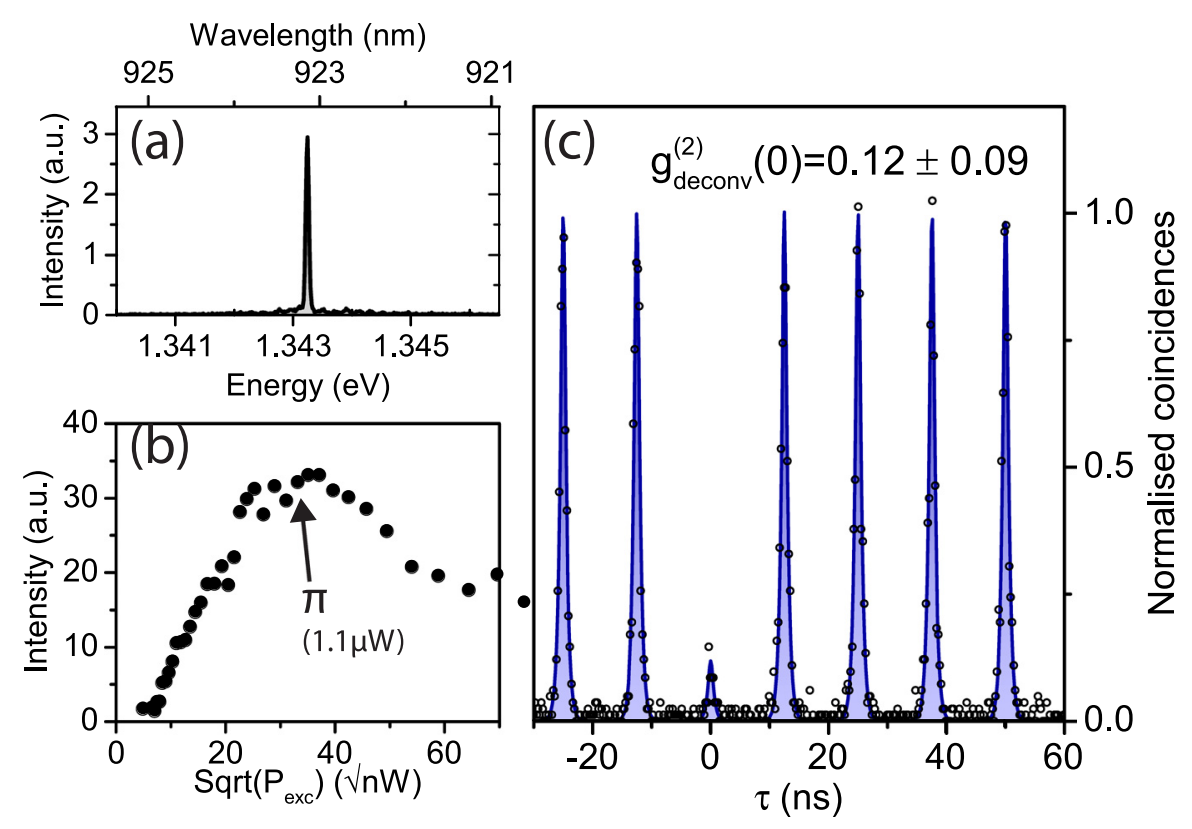

FIG. 4. (a) Resonance fluorescence emission spectrum of the quantum dot under $\pi$-pulse resonant excitation $(1 \mu \mathrm{W})$. (b) Intensity of the exciton emission as a function of the excitation power showing a Rabi-oscillation. The data point corresponding to the $\pi$-pulse area is indicated. (c) Photon autocorrelation measurement under $\pi$-pulse resonant excitation.

Interestingly, under $\pi$-pulse excitation, the QD exhibits an increased linewidth of about $45 \mu \mathrm{eV}$. Measuring the intensity of the QD emission as a function of the laser power, we observe a Rabi oscillation up to about a $2 \pi$ pulse area (cf. Fig. 4(b)) which is again a typical signature of the coherent control of the excitonic two-level system. The observed damping of the Rabi oscillation with increasing pulse area is probably caused by the coupling to longitudinal acoustic (LA) phonons. ${ }^{26,27}$

The excitation power dependent RF measurements allow us to determine the $\pi$-pulse condition for which we study the photon autocorrelation function. The corresponding data is displayed in Fig. 4(c), and the strong antibunching at zero time delay evidences single-photon emission from a single two-level system. A fit of the raw data with a convolution of an exponential function (emitter decay) with the Gaussian instrument response function (IRF) $(\Delta t=350 \mathrm{ps})$ yields a $g^{(2)}(0)$ value of 0.12 . We attribute to non-ideal value, imperfect laser-suppression caused by the wavelength dependence of the used quarter waveplate.

In conclusion, we have shown that site-controlled QDs grown by the buried-stressor growth technique exhibit promising optical properties under resonant excitation. We evidence the coherent interaction between exciton and laser field by observing the Mollow triplet sustained up to Rabi splittings of $200 \mu \mathrm{eV}$. Using resonant pulsed excitation, we prove single-photon emission by performing an intensity autocorrelation measurement obtaining $g^{(2)}(0)=0.12$. Our results demonstrate the high potential of this approach for the realization of scalable quantum devices based on high-quality singlephoton emitters. Further development could aim at dense arrays of site-controlled buried-stressor QDs and at their integration in microlenses for enhanced photon-extraction efficiency. The buried-stressor approach is also very suitable for the realization of micropillar lasers with a controlled number of QDs at the anti-node of the fundamental cavity mode.

The research leading to these results has received funding from the German Research Foundation via CRC 787 and Re2974/5-1, the Volkswagen Foundation via the project NeuroQNet and from the European Research Council under the European Union's Seventh Framework ERC Grant Agreement No. 615613.

${ }^{1}$ H. J. Kimble, Nature 453, 1023 (2008).

${ }^{2}$ N. Gisin and R. Thew, Nat. Photonics 1, 165 (2007).

${ }^{3}$ N. Sangouard, C. Simon, J. Minár, H. Zbinden, H. De Riedmatten, and N. Gisin, Phys. Rev. A 76, 050301(R) (2007).

${ }^{4}$ X. Ding, Y. He, Z. C. Duan, N. Gregersen, M. C. Chen, S. Unsleber, S. Maier, C. Schneider, M. Kamp, S. Höfling, C. Y. Lu, and J. W. Pan, Phys. Rev. Lett. 116, 020401 (2016).

${ }^{5}$ P. G. Kwiat, K. Mattle, H. Weinfurter, A. Zeilinger, A. V. Sergienko, and Y. Shih, Phys. Rev. Lett. 75, 4337 (1995).

${ }^{6}$ A. Dousse, L. Lanco, J. Suffczynski, E. Semenova, A. Miard, A. Lemaitre, I. Sagnes, C. Roblin, J. Bloch, and P. Senellart, Phys. Rev. Lett. 101, 267404 (2008).

${ }^{7}$ M. Gschrey, F. Gericke, A. Schüßler, R. Schmidt, J. H. Schulze, T. Heindel, S. Rodt, A. Strittmatter, and S. Reitzenstein, Appl. Phys. Lett. 102, 251113 (2013).

${ }^{8}$ A. Laucht, S. Pütz, T. Günthner, N. Hauke, R. Saive, S. Frédérick, M. Bichler, M.-C. Amann, A. W. Holleitner, M. Kaniber, and J. J. Finley, Phys. Rev. X 2, 011014 (2012).

${ }^{9}$ M. N. Makhonin, J. E. Dixon, R. J. Coles, B. Royall, I. J. Luxmoore, E. Clarke, M. Hugues, M. S. Skolnick, and A. M. Fox, Nano Lett. 14, 6997 (2014).

${ }^{10}$ E. Pelucchi, S. Watanabe, K. Leifer, Q. Zhu, B. Dwir, P. De Los Rios, and E. Kapon, Nano Lett. 7, 1282 (2007).

${ }^{11}$ A. Surrente, P. Gallo, M. Felici, B. Dwir, A. Rudra, and E. Kapon, Nanotechnology 20, 415205 (2009).

${ }^{12}$ C. Schneider, T. Heindel, A. Huggenberger, P. Weinmann, C. Kistner, M. Kamp, S. Reitzenstein, S. Höfling, and A. Forchel, Appl. Phys. Lett. 94, 111111 (2009).

${ }^{13}$ T. J. Pfau, A. Gushterov, J. P. Reithmaier, I. Cestier, G. Eisenstein, E. Linder, and D. Gershoni, Appl. Phys. Lett. 95, 243106 (2009).

${ }^{14}$ F. Albert, S. Stobbe, C. Schneider, T. Heindel, S. Reitzenstein, S. Höfling, P. Lodahl, L. Worschech, and A. Forchel, Appl. Phys. Lett. 96, 151102 (2010).

${ }^{15}$ K. D. Jöns, P. Atkinson, M. Müller, M. Heldmaier, S. M. Ulrich, O. G. Schmidt, and P. Michler, Nano Lett. 13, 126 (2013).

${ }^{16}$ S. Unsleber, S. Maier, D. P. S. McCutcheon, Y.-M. He, M. Dambach, M. Gschrey, N. Gregersen, J. Mørk, S. Reitzenstein, S. Höfling, C. Schneider, and M. Kamp, Optica 2, 1072 (2015).

${ }^{17}$ T. Huber, A. Predojevic, M. Khoshnegar, D. Dalacu, P. J. Poole, H. Majedi, and G. Weihs, Nano Lett. 14, 7107 (2014).

${ }^{18}$ W. Unrau, D. Quandt, J. H. Schulze, T. Heindel, T. D. Germann, O. Hitzemann, A. Strittmatter, S. Reitzenstein, U. W. Pohl, and D. Bimberg, Appl. Phys. Lett. 101, 211119 (2012).

${ }^{19}$ A. Strittmatter, A. Holzbecher, A. Schliwa, J. H. Schulze, D. Quandt, T. D. Germann, A. Dreismann, O. Hitzemann, E. Stock, I. A. Ostapenko, 
S. Rodt, W. Unrau, U. W. Pohl, A. Hoffmann, D. Bimberg, and V. Haisler, Phys. Status Solidi A: Appl. Mater. Sci. 209, 2411 (2012).

${ }^{20}$ A. Strittmatter, A. Schliwa, J. H. Schulze, T. D. Germann, A. Dreismann, O. Hitzemann, E. Stock, I. A. Ostapenko, S. Rodt, W. Unrau, U. W. Pohl, A. Hoffmann, D. Bimberg, and V. Haisler, Appl. Phys. Lett. 100, 093111 (2012).

${ }^{21}$ A. V. Kuhlmann, J. H. Prechtel, J. Houel, A. Ludwig, D. Reuter, A. D. Wieck, and R. J. Warburton, Nat. Commun. 6, 8204 (2015).

${ }^{22}$ H. Wang, Z. C. Duan, Y. H. Li, S. Chen, J. P. Li, Y. M. He, M. C. Chen, Y. He, X. Ding, C. Z. Peng, C. Schneider, M. Kamp, S. Höfling, C. Y. Lu, and J. W. Pan, Phys. Rev. Lett. 116, 213601 (2016).
${ }^{23}$ R. Trotta, J. S. Wildmann, E. Zallo, O. G. Schmidt, and A. Rastelli, Nano Lett. 14, 3439 (2014).

${ }^{24}$ Y.-J. Wei, Y. He, Y.-M. He, C.-Y. Lu, J.-W. Pan, C. Schneider, M. Kamp, S. Höfling, D. P. McCutcheon, and A. Nazir, Phys. Rev. Lett. 113, 097401 (2014).

${ }^{25}$ S. M. Ulrich, S. Ates, S. Reitzenstein, A. Löffler, A. Forchel, and P. Michler, Phys. Rev. Lett. 106, 247402 (2011).

${ }^{26}$ J. Förstner, C. Weber, J. Danckwerts, and A. Knorr, Phys. Rev. Lett. 91, 127401 (2003).

${ }^{27}$ A. J. Ramsay, A. V. Gopal, E. M. Gauger, A. Nazir, B. W. Lovett, A. M. Fox, and M. S. Skolnick, Phys. Rev. Lett. 104, 017402 (2010). 